\title{
Five times sit-to-stand test for ambulatory individuals with spinal cord injury: a psychometric study on the effects of arm placements
}

\author{
Lalita Khuna $\mathbb{B}^{1,2} \cdot$ Thiwabhorn Thaweewannakij ${ }^{1,2} \cdot$ Pattra Wattanapan $^{2,3} \cdot$ Pipatana Amatachaya $^{2,4}$. \\ Sugalya Amatachaya ${ }^{1,2}$
}

Received: 26 June 2019 / Revised: 14 September 2019 / Accepted: 20 September 2019 / Published online: 29 October 2019

(c) The Author(s), under exclusive licence to International Spinal Cord Society 2019

\begin{abstract}
Study design A psychometric study.

Objectives To investigate the reliability and the concurrent validity of the five times sit-to-stand test (FTSST) during various arm placement conditions in ambulatory participants with spinal cord injury (SCI). In addition, the difficulty of the FTSST during various arm placement conditions was compared using the number, characteristics, and physical ability of those who could complete the conditions.

Setting Rehabilitation centers and communities in Thailand.

Methods Eighty-two participants were assessed for their physical ability and ability to complete the FTSST in four arm placement conditions, namely, arms on a walking device, arms on knees, arms free by sides, and arms crossed over the chest, according to their ability and in random order.

Results The outcomes for all the FTSST conditions had excellent rater and test-retest reliability with a modest to strong correlation with their physical ability $(\rho=-0.39$ to -0.72$)$, and it is especially high for the condition with arms by sides. All participants could complete the conditions with arms on a walking device, and two-thirds of them could execute the other conditions. The physical ability of these individuals was significantly better, with the number of those with mild lesion severity being greater than those who could execute the FTSST with arms on a walking device $(p<0.01)$.

Conclusions Arm placements affected the psychometric properties and the ability to complete the FTSST of the participants. The current findings recommend the FTSST for ambulatory individuals with SCI using arms free by sides.
\end{abstract}

\section{Introduction}

The sit-to-stand ability is a basic but mechanically demanding activity that requires large joint torques, lower limb muscle force, balance ability, sensorimotor functions,

$\triangle$ Sugalya Amatachaya

samata@kku.ac.th

1 School of Physical Therapy, Faculty of Associated Medical Sciences, Khon Kaen University, Khon Kaen, Thailand

2 Improvement of Physical Performance and Quality of Life (IPQ) Research Group, Khon Kaen University, Khon Kaen, Thailand

3 Department of Rehabilitation Medicine, Faculty of Medicine, Khon Kaen University, Khon Kaen, Thailand

4 Department of Mechanical Engineering, Faculty of Engineering and Architecture, Rajamangala University of Technology Isan, Nakhon Ratchasima, Thailand and psychological factors [1]. Therefore, it is commonly incorporated into rehabilitation treatments and is used as an important clinical test to reflect lower limb motor strength (LEMS) and the dynamic balance control ability of many individuals with brain function disorders or other physical impairments [1-6].

Among the various methods of sit-to-stand tests, the five times sit-to-stand test (FTSST) is the most widely used and documented for its psychometric properties [1-7]. Nonetheless, the ability to perform FTSST can be affected by many factors, particularly chair seat height, foot placements, and arm positions [8]. There is clear evidence of the two former factors in the literature, in which a standard chair seat height was used $(43-46 \mathrm{~cm})$ [9] and the feet were placed with the heels at $10 \mathrm{~cm}$ behind the knees $[10,11]$. However, the arm positions vary in existing reports, including arms crossed over the chest [2, 4], arms free by the sides [12], arms on the thighs/knees [3], and arms on armrests/walking device $[12,13]$. The outcomes of these 
conditions showed no clear differences among healthy individuals who had adequate LEMS; thus they did not need addition momentum from their arms [13, 14].

Recently, the FTSST has been applied in ambulatory individuals with spinal cord injury (SCI) who have intact brain functions but with various degrees of bilateral sensorimotor deterioration affecting their LEMS, balance ability, and risk of fall $[15,16]$. However, the existing reports applied different arm placements for the FTSST, i.e., arms free by the sides or arms on a walking device $[11,17,18]$, whereas a standard protocol suggests the use of arms folded over the chest [10], without clear evidence confirming the psychometric properties for clinical implication of these arm placement conditions in these individuals. It has been reported that the positions of the arms affect the body's center of mass, body balance, and the ease of performing the tasks $[14,19]$. For example, a forward arm position promotes the forward momentum needed for transferring the body's center of mass effectively during rising from a chair [19]. On the contrary, restriction of the arms with the body while rising from sitting leads to the use of different strategy, particularly ankle angular displacement patterns [14]. Consequently, the researchers hypothesized that different arm placements would affect the psychometric properties of the FTSST due to the task demands of each condition, where we expected that the condition with arms on a walking device would be the easiest condition and the one with arms crossed over the chest would be the most difficult condition for ambulatory individuals with SCI. Therefore, this study explored the psychometric properties, including the reliability (rater and test-retest reliability) and concurrent validity (the correlation between the outcomes of FTSST and physical ability tests), of the FTSST using different arm placement conditions, namely, arms on a walking device, arms on knees, arms free by the sides, and arms crossed over the chest. In addition, this study compared the difficulty among the conditions using the number, characteristics, and physical ability of ambulatory individuals with SCI who could complete each condition.

\section{Methods}

\section{Participants}

This study examined the psychometric properties of the FTSST in ambulatory participants with incomplete SCI from rehabilitation centers and communities. The eligible participants should be at least 18 years old, have a body mass index of $18.5-29.9 \mathrm{~kg} / \mathrm{m}^{2}$, have motor-incomplete SCI as determined by the International Standards for Neurological Classification of SCI (ISNCSCI) or the
American Spinal Cord Injury Association Impairment Scale (AIS C and D), as well as those with cauda equina syndromes [15]. The eligible participants also had the ability of independent standing up from a chair with or without using their hands and walking with or without a walking device over at least $10 \mathrm{~m}$. The exclusion criteria were any signs and symptoms that might affect their ability to perform the tests, such as pain in the muscles or joints with an intensity of pain more than 5 out of 10 on a numeric pain-rating scale, joint deformities affecting mobility, and other neurological disorders. The sample size for a reliability study was derived from previous studies (20-30 participants) [20, 21]. The sample size for a concurrent validity study was calculated from a pilot study $(n=40)$, which sets the level of correlation $(r)$ to $0.32-0.41$ for each physical ability test and the $\alpha$ to 0.05 , the findings indicated this study required $48-82$ participants. The experimental protocols of the study were approved by the local ethics committee (HE 601349). The eligible participants signed an informed consent document prior to participation in the study.

\section{Research protocol}

The eligible participants were interviewed and assessed for their demographics and SCI characteristics, including cause of SCI, post-injury time, and level and severity of injury according to the ISNCSCI [15]. Then, the participants were assessed for their ability to perform FTSST for each arm placement condition in random order with video recording to further analyze the reliability data. On the following day, the participants were assessed for their LEMS of the eight major muscle groups and functional tests to reflect their physical ability relating to levels of independence of participants [22-24] who could complete FTSST in each arm placement condition and to verify the concurrent validity of the FTSST conditions. The details of the tests are as follows:

\section{FTSST}

The participants were seated on a standard armless chair with their back upright against the backrest of the chair, hip flexion at $90^{\circ}$, and their feet flat on the floor with the heels at $10 \mathrm{~cm}$ behind their knees. The tests recorded the time required to complete five chair-rise cycles in the fastest and safest manner possible while placing their arms in the following four conditions (Fig. 1a-d):

(1) Arms on a walking device (a standard walker) $[12,13]$

(2) Arms on the knees [3]

(3) Arms hanging freely at their sides [12]

(4) Arms folded over the chest $[2,4]$ 


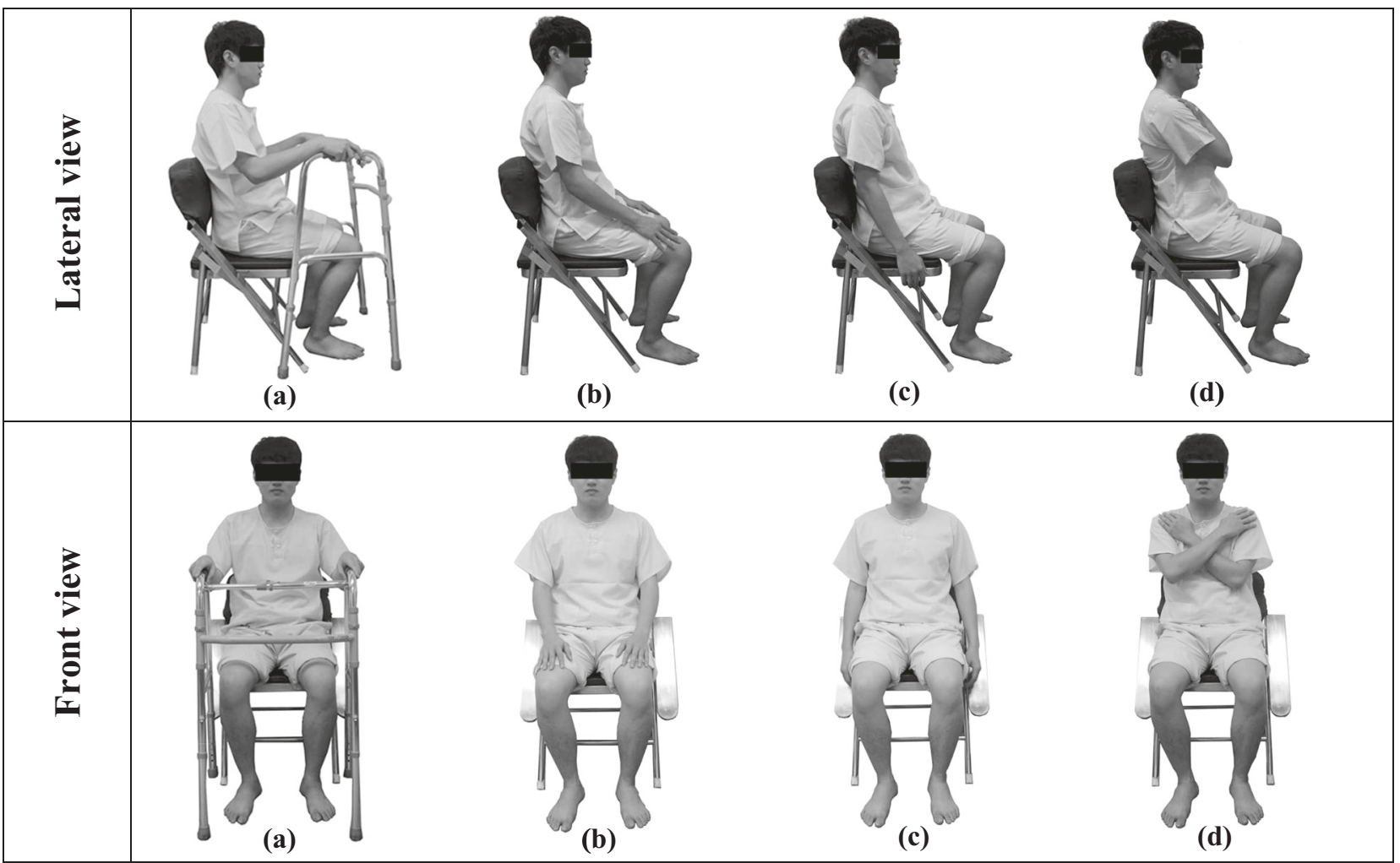

Fig. 1 Arm placement conditions for the five times sit-to-stand test. a Arms on a walking device. b Arms on knees. c Arms by sides. d Arms crossed over the chest

The participants performed these conditions according to their own ability in random order and without compulsion to complete all tests if they were unable to do to reflect the difficulty of each condition. The average time required from the command "go" to the participants' backs touching the backrest of the chair in the fifth repetition over the three trials and the number of participants who could complete the conditions were recorded $[3,12]$.

\section{LEMS}

The participants were assessed for the strength of their eight major lower limb muscles, namely, hip flexors, hip extensors, hip abductors, hip adductors, knee flexors, knee extensors, ankle dorsiflexors, and ankle plantar flexors, using the manual muscle test [25]. The strength of each muscle group was graded on a 12-point scale (i.e., 0, 1, 2-, $2,2+, 3-, 3,3+, 4-, 4,4+$, and 5), and the outcomes were converted into scores from 0 to 11 [25]. The total LEMS in this study was 176 scores.

\section{Functional tests}

The participants were assessed for their functional ability using the 10-meter walk test (10MWT), timed up-and-go test (TUGT), and 6-minute walk test (6MWT) to represent the overall quality of gait and the ability of community participation [12, 26], dynamic balance control ability and fall risk, and functional endurance [22, 23], respectively. Their walking ability and the requirement of walking devices and/or external assistance while performing the 10MWT were also used to indicate the Walking Index for Spinal Cord Injury (WISCI) II scores [23, 27] of the participants. During the tests, the participants were fastened with a lightweight safety belt around their waist, and a physical therapist was always by their side to ensure their safety and the accuracy of the tests.

\section{Reliability tests}

The first 30 participants were assessed by an expert health professional (i.e., a physical therapist who had experience using the FTSST for at least 3 years) and a novice health professional to represent the reliability of the FTSST [20]. The details of the reliability assessments are as follows:

Rater reliability: A novice rater was trained in the methods for administering the tests and recording the outcomes, with practice sessions of about $30 \mathrm{~min}$. Both raters recorded the outcomes of FTSST of participants who could complete each condition from the video recording, with a 1-week interval between the first and 
second sessions. The data from both sessions of each rater were used to analyze the intra-rater reliability, and those from the first session of both raters were used to reflect the inter-rater reliability [3].

Test-retest reliability: An expert rater recorded the ability of the first 30 participants who were able to perform each condition. Seven days later, the same 30 participants were re-assessed for the outcomes of the FTSST. The data from the first and second assessments were used to represent the test-retest reliability [3, 4].

\section{Data analysis}

Descriptive statistics were used to explain the demographics and the SCI characteristics of participants as well as the findings of the study. The Shapiro-Wilk test was used to estimate the normality of the data. The intraclass correlation coefficients $\left(\mathrm{ICC}_{3,3}\right.$ : the raters were only the raters of interest, not randomly selected, and the reliability was calculated using the average data over three trials [28]) were employed to estimate the reliability of the FTSST conditions. The ICC values of 0.75 and higher were considered excellent, those $<0.40$ were poor, and those between these ranges had moderate-to-good reliability [29]. Portney and Watkins [30] suggested that ICCs for clinical tests should be at least 0.90 to obtain reliable outcomes. The ICC data of the test-retest reliability were used to calculate (1) the standard error of measurement (SEM), which is used to estimate the expected error in interpreting an individual's test score [31], using the formula $\mathrm{SEM}=(\mathrm{SD}) \times \sqrt{ }(1-\mathrm{ICC})$, where $\mathrm{SD}$ is the standard deviation of the two sessions of the FTSST trials in the same participants [31]), and (2) the minimal detectable change (MDC), which is the estimated smallest change in the score that could be detected beyond the measurement errors, using the formula MDC $=1.96 \times \mathrm{SEM} \times \sqrt{2}$ for each FTSST condition [32]. The Pearson's correlation coefficients $(r)$ or the Spearman rank correlation coefficients $(\rho)$ were utilized to explain the levels of correlation (concurrent validity) of the FTSST and the physical ability data. The strength of the correlation was defined as modest ( $r$ or $\rho$ of $0.30-0.49$ ), moderate ( $r$ or $\rho=0.50-0.69)$, and strong $(r$ or $\rho>0.70)$ [33]. In addition, the one-way ANOVA or the Kruskal-Wallis test was used to compare the continuous data of the participants who could complete the FTSST conditions, and the Bonferroni post hoc or the Mann-Whitney $U$ test was used to identify the pairwise differences. The level of significant differences was set to $p<0.05$.

\section{Results}

Eighty-two participants with incomplete SCI were involved in this study, and the first 30 participants also participated in the reliability study (Table 1). All participants were able to complete the FTSST with arms on a walking device, and $65 \%(n=52-54$, Table 1) could accomplish the other conditions $(p<0.001)$. The participants considered the FTSST with arms crossed over the chest as the most difficult condition and that with the arms on a walking device as the easiest one. The participants who completed the condition with arms on a walking device had the number of

Table 1 Demographic and spinal cord injury characteristics of the participants who completed the validity and reliability study

\begin{tabular}{|c|c|c|c|c|c|}
\hline \multirow[t]{2}{*}{ Variable } & \multicolumn{5}{|l|}{ Concurrent validity study } \\
\hline & $\begin{array}{l}\text { Arms on a walking device } \\
(n=82)\end{array}$ & $\begin{array}{l}\text { Arms on knee } \\
(n=54)\end{array}$ & $\begin{array}{l}\text { Arms by sides } \\
(n=53)\end{array}$ & $\begin{array}{l}\text { Arms crossed over the } \\
\text { chest }(n=52)\end{array}$ & $\begin{array}{l}\text { Reliability tests } \\
(n=30)\end{array}$ \\
\hline Age $(\text { years })^{a}$ & $52.0 \pm 14.2(48.9-55.1)$ & $50.5 \pm 13.3(46.8-54.2)$ & $50.3 \pm 13.3(46.6-54.0)$ & $50.3 \pm 13.5(46.5-54.0)$ & $51.1 \pm 13.4(46.1-56.1)$ \\
\hline Body mass index $\left(\mathrm{kg} / \mathrm{m}^{2}\right)^{\mathrm{a}}$ & $22.6 \pm 3.5(21.8-23.3)$ & $22.8 \pm 3.8(21.7-23.8)$ & $22.7 \pm 3.8(21.6-23.8)$ & $22.7 \pm 3.7(21.6-23.8)$ & $23.5 \pm 3.6(22.2-24.9)$ \\
\hline Post-injury time (months) ${ }^{\mathrm{a}}$ & $89.8 \pm 79.3(69.3-104.2)$ & $85.1 \pm 80.5(63.1-107.1)$ & $86.0 \pm 81.0(63.7-108.4)$ & $87.2 \pm 81.4(64.5-109.8)$ & $89.9 \pm 80.7(59.8-120.1)$ \\
\hline Gender: male $\mathrm{e}^{\mathrm{b}, \mathrm{c}}$ & $69(84)$ & $47(87)$ & $46(87)$ & $45(87)$ & $26(87)$ \\
\hline Stage of injury: chronic ${ }^{b, c}$ & $74(90)$ & $48(89)$ & $47(89)$ & $46(88)$ & $28(93)$ \\
\hline Cause of injury: non-traumatic ${ }^{\mathrm{b}, \mathrm{c}}$ & $41(50)$ & $25(46)$ & $24(45)$ & $24(46)$ & $19(63)$ \\
\hline Level of injury: incomplete paraplegia ${ }^{b, c}$ & $55(67)$ & $33(61)$ & $32(60)$ & $32(62)$ & $20(67)$ \\
\hline AIS: $\mathrm{D}^{\mathrm{b}, \mathrm{c}^{*}}$ & $63(77)$ & $50(93)$ & $49(92)$ & $48(92)$ & $20(67)$ \\
\hline \multicolumn{6}{|l|}{ Using a walking device ${ }^{b}$} \\
\hline Walker & $31(38)$ & $11(20)$ & $11(21)$ & $10(19)$ & $10(33)$ \\
\hline Crutches & $8(10)$ & $2(4)$ & $2(4)$ & $2(4)$ & $2(7)$ \\
\hline Cane & $14(17)$ & $12(22)$ & $11(21)$ & $11(21)$ & $8(27)$ \\
\hline None & $29(35)$ & $29(54)$ & $29(54)$ & $29(56)$ & $10(33)$ \\
\hline
\end{tabular}

Data comparisons among those who completed each arm placement condition were performed using one-way ANOVA for continuous data and $\chi^{2}$ test for categories data, * indicated significant differences among the conditions $(p=0.008)$

AIS American Spinal Injury Association Impairment Scale

${ }^{a}$ Data are presented using mean \pm standard deviation (95\% confidence interval)

${ }^{\mathrm{b}}$ Data are presented using $n(\%)$

${ }^{\mathrm{c}}$ These variables were categorized according to the following criteria: Gender: male/female; Cause: non-traumatic/traumatic; Level of injury: incomplete paraplegia/incomplete tetraplegia; Stage of injury: sub-acute $(\leq 12$ months)/chronic ( $>12$ months); AIS class: C/D 
Fig. 2 Data of five times sit-tostand test, lower extremity motor strength, and functional ability tests of participants who could complete in each arm placement condition. Note: Total score of LEMS $=176$, total score of WISCI II $=20$, data were analyzed using Kruskal-Wallis test, * indicated the pairwise differences using the Mann-Whitney $U$ test ( $p$-value $<0.05$ )

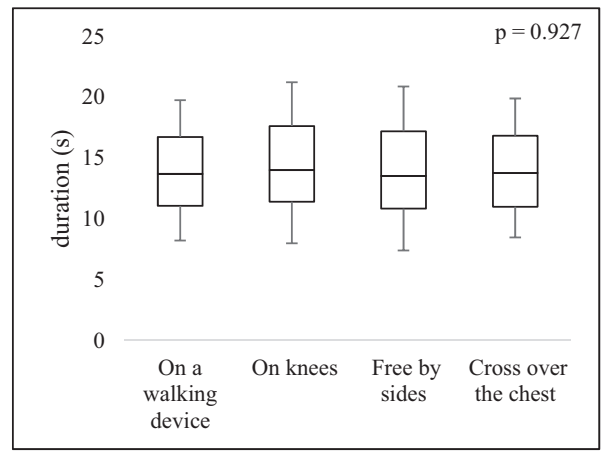

(a) Five Times Sit-to-Stand Test

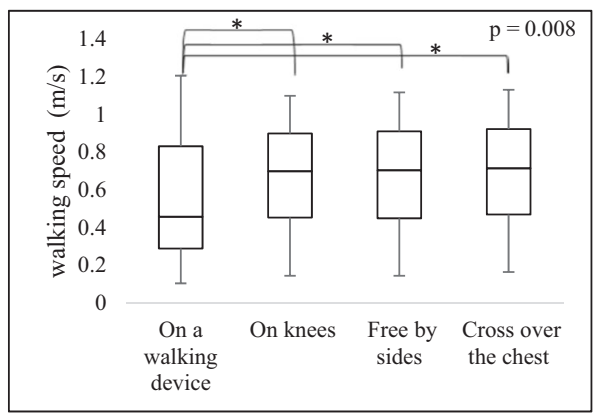

(c) 10-Meter Walk Test

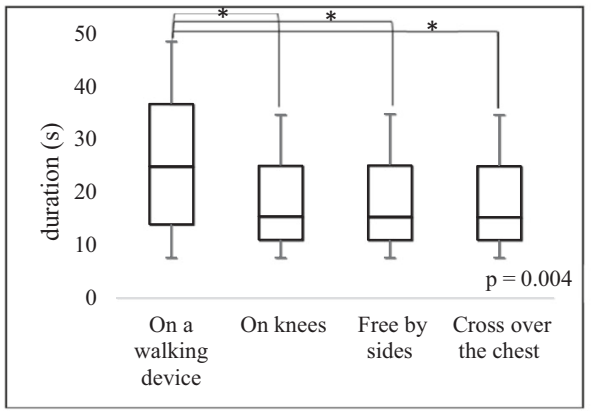

(e) Time Up and Go Test

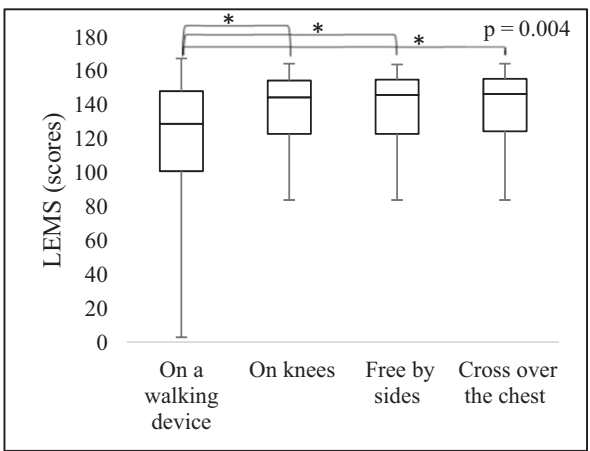

(b) Lower extremity motor strength (LEMS)

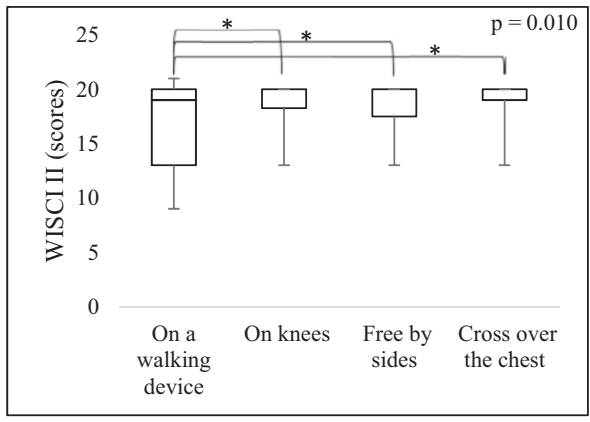

(d) Walking Index for Spinal Cord Injury II

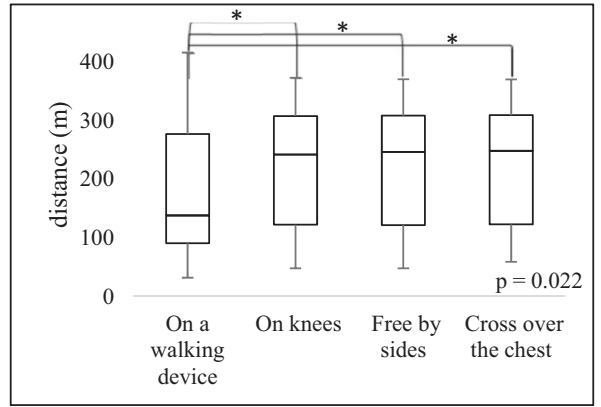

(f) 6-Minute Walk Test those with AIS D significantly less than those who were able to perform the other conditions $(p=0.008$, Table 1$)$. However, there were no significant differences in the time to complete the FTSST conditions (Fig. 2a). On the contrary, the LEMS and functional ability of the participants who could accomplish the FTSST with arms on a walking device were significantly poorer than those of the participants who could complete the other three conditions $(p<$ 0.05 ), and there was no significant difference among these three conditions $(p>0.05$, Fig. $2 b-f)$.

\section{Reliability of the FTSST}

All 30 participants could complete the condition with arms on a walking device, and 20-21 participants were able to perform the other three conditions (Table 2). The outcomes of all FTSST conditions showed excellent intra- and inter-rater reliability for both the expert and novice raters $\left(\mathrm{ICC}_{3,3}=0.998-1.000\right.$; Table 2). Every condition had an excellent test-retest reliability $\left(\mathrm{ICC}_{3,3}=0.956-0.989\right)$ with a small amount of SEM and MDC. In comparing the conditions, testing with arms by the sides had the smallest values of SEM and MDC, and the condition with arms on a walking device had the largest values of SEM and MDC (Table 2).

\section{Concurrent validity of the FTSST}

The time to complete the FTSST of all conditions was significantly correlated with LEMS and all the functional measures, particularly for 10MWT, TUGT, and 6MWT ( $\rho=-0.39$ to $-0.72, p<0.01$ to $p<0.001$, Table 3 ). In 
Table 2 Rater and test-retest reliability, standard error of measurement (SEM), and minimal detectable change (MDC) of the five times sit-to-stand test (FTSST) of the four arm placement conditions

\begin{tabular}{|c|c|c|c|c|}
\hline Conditions of the FTSST & $\begin{array}{l}\text { Arms on a walking } \\
\text { device }(n=30)\end{array}$ & $\begin{array}{l}\text { Arms on knees } \\
(\mathrm{n}=21)\end{array}$ & $\begin{array}{l}\text { Arms by sides } \\
(n=20)\end{array}$ & $\begin{array}{l}\text { Arms crossed over } \\
\text { the chest }(n=20)\end{array}$ \\
\hline \multicolumn{5}{|l|}{ Rater reliability } \\
\hline \multicolumn{5}{|l|}{ Expert rater } \\
\hline \multicolumn{5}{|c|}{ - Duration of the FTSST ${ }^{\mathrm{a}}$ (second) } \\
\hline Session 1 & $15.3(5.10)$ & $15.7(5.16)$ & $16.0(5.34)$ & $15.5(4.54)$ \\
\hline Session 2 & $15.4(5.12)$ & $15.8(5.22)$ & $16.1(5.45)$ & $15.9(4.33)$ \\
\hline - Intra-rater reliability ${ }^{\mathrm{b}}$ & $1.000(0.999-1.000)$ & $0.999(0.998-1.000)$ & $0.999(0.997-1.000)$ & $1.000(0.999-1.000)$ \\
\hline \multicolumn{5}{|l|}{ Novice rater } \\
\hline \multicolumn{5}{|c|}{ - Duration of the FTSST ${ }^{\mathrm{a}}$ (second) } \\
\hline Session 1 & $15.2(5.05)$ & $15.7(5.33)$ & $16.0(5.47)$ & $15.4(4.51)$ \\
\hline Session 2 & $15.6(5.16)$ & $16.0(5.33)$ & $16.1(5.40)$ & $15.6(4.61)$ \\
\hline - Intra-rater reliability ${ }^{\mathrm{b}}$ & $0.998(0.973-0.999)$ & $0.999(0.994-1.000)$ & $0.999(0.998-1.000)$ & $1.000(0.999-1.000)$ \\
\hline - Inter-rater reliability ${ }^{\mathrm{b}}$ & $0.999(0.995-0.999)$ & $0.998(0.995-0.999)$ & $1.000(0.999-1.000)$ & $1.000(0.999-1.000)$ \\
\hline \multicolumn{5}{|l|}{ Test-retest reliability } \\
\hline \multicolumn{5}{|c|}{ - Duration of the FTSST ${ }^{\mathrm{a}}$ (second) } \\
\hline Session 1 & $15.7(5.15)$ & $16.1(5.71)$ & $16.0(5.18)$ & $16.2(5.97)$ \\
\hline Session 2 & $14.8(5.27)$ & $15.3(5.53)$ & $15.2(5.48)$ & $15.2(4.95)$ \\
\hline - Test-retest reliability ${ }^{\mathrm{b}}$ & $0.956(0.907-0.979)$ & $0.975(0.939-0.990)$ & $0.989(0.971-0.996)$ & $0.962(0.905-0.985)$ \\
\hline - SEM & 1.09 & 0.88 & 0.55 & 1.05 \\
\hline - MDC & 3.01 & 2.44 & 1.53 & 2.93 \\
\hline
\end{tabular}

${ }^{a}$ Durations of the FTSST were presented by mean (standard deviation)

${ }^{b}$ Reliability data were presented using the intraclass correlation coefficients $\left(\mathrm{ICC}_{3,3}\right)$ with $95 \%$ confidence intervals

Table 3 The correlation between the four arm placement conditions of the five times sitto-stand test (FTSST) and physical ability tests

\begin{tabular}{llllll}
\hline Conditions of the FTSST & LEMS & 10MWT & WISCI II & TUGT & 6MWT \\
\hline Arms on a walking device $(n=82)$ & $-0.43^{* * *}$ & $-0.55^{* * *}$ & $-0.44^{* * *}$ & $0.60^{* * *}$ & $-0.63^{* * *}$ \\
Arms on knees $(n=54)$ & $-0.40^{* *}$ & $-0.62^{* * *}$ & $-0.49^{* * *}$ & $0.70^{* * *}$ & $-0.70^{* * *}$ \\
Arms by sides $(n=53)$ & $-0.43^{* * *}$ & $-0.65^{* * *}$ & $-0.53^{* * *}$ & $0.71^{* * *}$ & $-0.72^{* * *}$ \\
Arms crossed over the chest $(n=52)$ & $-0.39^{* *}$ & $-0.59^{* * *}$ & $-0.50^{* * *}$ & $0.67^{* * *}$ & $-0.67^{* * * *}$ \\
\hline
\end{tabular}

The data are presented using Spearman's rho coefficient $(\rho)$

LEMS lower extremity motor strength, 10MWT 10-meter walk test, WISCI II walking index for spinal cord injury II, TUGT timed up and go test, $6 M W T$ 6-minute walk test

${ }^{* *} p<0.01,{ }^{* * *} p<0.001$ comparing the FTSST conditions, the tests conducted with arms by the sides had the highest correlation with LEMS and functional ability $(\rho=-0.43$ to $-0.72 ; p<0.001)$, and the test with arms on a walking device showed a slightly lower correlation than those of the other conditions $(\rho=-0.43$ to $-0.63 ; p<0.01$, Table 3$)$.

\section{Discussion}

This study explored the influence of arm placements on the psychometric properties and difficulty of the FTSST among the four arm placement conditions in ambulatory individuals with SCI. All participants could complete the FTSST with arms on a walking device, and only two-thirds could perform the other three conditions $(p<0.001$, Table 1$)$. The time to complete the FTSST showed modest to strong correlation with LEMS and functional ability, with excellent reliability and small values of SEM and MDC, particularly the condition with arms by the sides (Tables 2 and 3).

The LEMS and functional ability of participants with SCI who completed the conditions with arms on a walking device were significantly poorer than those of the participants who could perform the other conditions, thus suggesting that this condition was the easiest one (Fig. 2b-f). Upper limb placing on a walking device promoted forward momentum for body-weight transferring and the ease of the tasks, and helped the participants to complete the test with a 
similar amount of time as that of the other conditions $(p>$ 0.05, Fig. 2a). Arborelius et al. [34] reported that using the arms reduces hip moments by about $50 \%$ of the extension moment needed for a sit-to-stand task. Alexander et al. [35] found that the proportion of older adults who were unable to rise from a chair decreased from $32 \%$ to only $1 \%$ when hand use was allowed. However, various amounts of upper limb contribution across the trials while rising resulted in the widest range of SEM and MDC data in comparison with the other conditions (Table 2).

By contrast, the other three conditions required the most contribution from the lower extremities. Although the condition with arms on knees enabled the participants to use their arms, the sensorimotor impairments of the lower limbs could limit the participants to forcefully push their legs while rising up. Therefore, this condition still relied mostly on the lower limb functions, while the body base of support was clearly reduced in comparison with the condition of using a walking device. Consequently, the number of participants who completed this condition was similar to that of the conditions with arms by the sides and arms crossed over the chest (Table 1). The condition with arms crossed over the chest also limited the upper limb contribution for body balance while risen the body center of mass, which challenged the balance ability. Thus, the participants with bilateral sensorimotor impairments were unable to perform to their optimal ability in this condition. These assumptions were associated with the suggestion from the participants that performing the FTSST with arms crossed over the chest was the most difficult condition and that with arms on a device was the easiest. Carr [19] also reports that restriction of the arms with the body while standing up leads to a different angular displacement of the ankles and significantly lengthened the time required to produce the maximum support moment than that with the arms free. By contrast, the condition with arms hanging naturally by the sides afforded the body balance, and enabled the participants to perform the test optimally. Thus, the reliability and validity data were the highest in the condition with arms free by sides (Tables 2 and 3).

The higher correlation between the outcomes from FTSST with the 10MWT, TUGT, and 6MWT data $(\rho=$ -0.55 to -0.72 ) as compared to that with the LEMS and WISCI II ( $\rho=-0.39$ to -0.53 ; Table 3$)$ suggested the characteristics of the tests. The LEMS reflected the system functions (i.e., only lower limb muscle strength) that was assessed in a lying or sitting position, and the outcomes of LEMS and WISCI II were in ordinal scales [25, 27]. Conversely, the outcomes of the 10MWT, TUGT, and 6MWT were the mobility tests that required the contributions of many body systems and provided continuous data, similar to the FTSST. Such characteristics may enhance the levels of correlation in comparison with the LEMS and WISCI II scores (Table 3). This assumption was associated with the data on the participants with chronic stroke, which found a high correlation $(\rho=-0.753$ to -0.830$)$ when muscle strength was reported using continuous data from a handheld dynamometer [3].

The excellent rater and test-retest reliability could be related to the characteristics of the FTSST that were timed from the command "go" until the participants' backs were against the backrest of the chair after the fifth repetition of sit-to-stand cycles. [3, 12]. Such criteria clearly determined the time recorded by the health professionals. Furthermore, the measurement for the outcomes from the video recording reduced the variation in the participants' performance. All of the procedures enhanced and confirmed the reliability of the outcomes of FTSST for clinical trials (more than 0.90) [30]. The findings were consistent with those of Mong et al. [3], who used the same timing protocols and also found an excellent reliability of the FTSST in people with chronic stroke (ICC $=0.970-0.999$ ). By contrast, a study on individuals with Parkinson's disease that recorded the time from the command "go" to the participants' buttocks contacting with the seat after the fifth repetition found a wide range of ICC data (0.76-0.99) [4].

The excellent test-retest reliability of the FTSST conditions was further confirmed by the small SEM and MDC values. The SEM is an index for the absolute reliability of a test, in which a small SEM indicates high absolute reliability, that is, the recorded score is close to the true score [30]. The MDC reflects the smallest change in the score beyond the measurement errors, that is, a true change in performance with $95 \%$ confidence [32]. Previous data on patients with Alzheimer's disease [5] and on older female adults [7] reported a similar range of SEM $(0.9-1.39 \mathrm{~s})$ and MDC (2.5- $2.73 \mathrm{~s})$.

Previous studies suggested the test of FTSST with arms on knees and arms crossed over the chest to be used in other populations [1-4]. The current findings suggested that various arm placements could be used to report the outcomes of the FTSST and that the condition with arms by the sides would be optimal for ambulatory individuals with SCI. However, some limitations of the study must be addressed. The number of participants in each FTSST condition was not equal to reflect the natural characteristics and the difficulty of the tests. Nonetheless, $65 \%$ of participants had rather good functional ability because they could complete all FTSST conditions. Thus, this may confound validity of the condition with arms on a walking device, and the current findings might not reflect the data on those who could complete only the test with arms on a walking device. In addition, the findings may be confounded by various degrees of lower limb loading and upper limb contribution on a walking device or knees. Furthermore, the other psychometric properties of the 
FTSST in individuals with SCI are not available. Therefore, further studies that measured lower limb and upper limb loading during sit-to-stand with data comparisons between those who perform the FTSST with or without hands, and reporting data on other psychometric properties are needed to extend the clinical implication of the FTSST particularly to these individuals.

\section{Conclusion}

All of the FTSST conditions used in this study showed excellent rater and test-retest reliability, with a modest to strong correlation with the physical ability of the participants. In all the conditions, the condition with arms by the sides offered the best psychometric properties for clinical and research works in ambulatory individuals with SCI.

\section{Data availability}

All data generated and analyzed in this study are available from the corresponding author on request.

Funding This work was supported by the Royal Golden Jubilee PhD Program (PHD58K0223) and the Improvement of Physical Performance and Quality of Life (IPQ) research groups, Khon Kaen University, Khon Kaen, Thailand.

Author contributions All authors were responsible for research conception and design, critical revision of the article for important intellectual content, provision of study materials or patients. LK was additionally involved in the data acquisition, statistical analysis, and drafting a manuscript. SA was also responsible for the project management, funding application, and finalizing the manuscript.

\section{Compliance with ethical standards}

Conflict of interest The authors declare that they have no conflict of interest.

Statement of ethics We certify that all applicable institutional and governmental regulations concerning the ethical use of human volunteers were followed during the course of this research.

Publisher's note Springer Nature remains neutral with regard to jurisdictional claims in published maps and institutional affiliations.

\section{References}

1. Lord SR, Murray SM, Chapman K, Munro B, Tiedemann A. Sitto-stand performance depends on sensation, speed, balance, and psychological status in addition to strength in older people. $\mathrm{J}$ Gerontol A Biol Sci Med Sci. 2002;57:M539-43.

2. Wang TH, Liao HF, Peng YC. Reliability and validity of the fiverepetition sit-to-stand test for children with cerebral palsy. Clin Rehabil. 2011;26:664-71.
3. Mong Y, Teo TW, Ng SS. 5-repetition sit-to-stand test in subjects with chronic stroke: reliability and validity. Arch Phys Med Rehabil. 2010;91:407-13.

4. Duncan RP, Leddy AL, Earhart GM. Five times sit-to-stand test performance in Parkinson's Disease. Arch Phys Med Rehabil. 2011;92:1431-6.

5. Suttanon P, Hill KD, Dodd KJ, Said CM. Retest reliability of balance and mobility measurements in people with mild to moderate Alzheimer's disease. Int Psychogeriatr. 2011;23:1152-9.

6. Meretta BM, Whitney SL, Marchetti GF, Sparto PJ, Muirhead RJ. The five times sit to stand test: responsiveness to change and concurrent validity in adults undergoing vestibular rehabilitation. $\mathbf{J}$ Vestib Res. 2006;16:233-43.

7. Goldberg A, Chavis M, Watkins J, Wilson T. The five-times-sitto-stand test: validity, reliability and detectable change in older females. Aging Clin Exp Res. 2012;24:339-44.

8. Janssen WG, Bussmann HB, Stam HJ. Determinants of the sit-tostand movement: a review. Phys Ther. 2002;82:866-79.

9. Bohannon RW. Reference values for the five-repetition sit-tostand test: a descriptive meta-analysis of data from elders. Percept Mot Skills. 2006;103:215-22.

10. Bohannon RW. Measurement of sit-to-stand among older adults. Top Geriatr Rehabil. 2012;28:11-6.

11. Shepherd RB, Koh HP. Some biomechanical consequences of varying foot placement in sit-to-stand in young women. Scand J Rehabil Med. 1996;28:79-88.

12. Poncumhak P, Saengsuwan J, Kumruecha W, Amatachaya S. Reliability and validity of three functional tests in ambulatory patients with spinal cord injury. Spinal Cord. 2013;51:214-7.

13. Etnyre B, Thomas DQ. Event standardization of sit-to-stand movements. Phys Ther. 2007;87:1651-66.

14. Ng SS, Kwong PW, Chau MS, Luk IC, Wan SS, Fong SS. Effect of arm position and foot placement on the five times sit-to-stand test completion times of female adults older than 50 years of age. $\mathrm{J}$ Phys Ther Sci. 2015;27:1755-9.

15. Kirshblum SC, Burns SP, Biering-Sorensen F, Donovan W, Graves DE, Jha A, et al. International standards for neurological classification of spinal cord injury (Revised 2011). J Spinal Cord Med. 2011;34:535-46.

16. Amatachaya S, Wannapakhe J, Arrayawichanon P, Siritarathiwat W, Wattanapun P. Functional abilities, incidences of complications and falls of patients with spinal cord injury 6 months after discharge. Spinal Cord. 2011;49:520-4.

17. Poncumhak P, Saengsuwan J, Amatachaya S. Ability of walking without a walking device in patients with spinal cord injury as determined using data from functional tests. J Spinal Cord Med. 2014;37:389-96.

18. Srisim K, Saengsuwan J, Amatachaya S. Functional assessments for predicting a risk of multiple falls in independent ambulatory patients with spinal cord injury. J Spinal Cord Med. 2015;38:439-45.

19. Carr JH. Balancing the centre of body mass during standing up. Physiother Theory Pract. 1992;8:159-64.

20. Morrow JR Jr, Jackson AW. How "significant" is your reliability? Res Q Exerc Sport. 1993;64:352-5.

21. Bohannon RW. Test-retest reliability of the five-repetition sit-tostand test: a systematic review of the literature involving adults. $\mathrm{J}$ Strength Cond Res. 2011;25:3205-7.

22. Lapointe R, Lajoie Y, Serresse O, Barbeau H. Functional community ambulation requirements in incomplete spinal cord injured subjects. Spinal Cord. 2001;39:327-35.

23. Jackson AB, Carnel CT, Ditunno JF, Read MS, Boninger ML, Schmeler MR, et al. Outcome measures for gait and ambulation in the spinal cord injury population. J Spinal Cord Med. 2008;31:487-99. 
24. Lam T, Noonan VK, Eng JJ. SCIRE Research Team. A systematic review of functional ambulation outcome measures in spinal cord injury. Spinal Cord. 2008;46:246-54.

25. Kendall FP, McCreary EK, Provance PG, editors. Muscle testing and function. 4th ed. Philadelphia: Lippincott, Williams and Wilkins; 1993.

26. van Hedel HJ, Wirz M, Dietz V. Assessing walking ability in subjects with spinal cord injury: validity and reliability of 3 walking tests. Arch Phys Med Rehabil. 2005;86:190-6.

27. Morganti B, Scivoletto G, Ditunno P, Ditunno JF, Molinari M. Walking index for spinal cord injury (WISCI): criterion validation. Spinal Cord. 2005;43:27-33.

28. Koo TK, Li MY. A guideline of selecting and reporting intraclass correlation coefficients for reliability research. J Chiropr Med. 2016;15:115-63.

29. Andresen EM. Criteria for assessing the tools of disability outcomes research. Arch Phys Med Rehabil. 2000;81:S15-20.
30. Portney L, Watkins M, editors. Foundations of clinical research applications to practice. 3rd ed. Upper Saddle River, New Jersey: Pearson Prentice Hall; 2009.

31. Bruton A, Conway JH, Holgate ST. Reliability: what is it, and how is it measured? Physiotherapy. 2000;29:94-9.

32. Beaton DE. Understanding the relevance of measured change through studies of responsiveness. Spine. 2000;25:3192-9.

33. Andresen EM, Fonts BS, Romeis JC, Brownson CA. Performance of health-related quality-of-life instruments in a spinal cord injured population. Arch Phys Med Rehabil. 1999;80:877-84.

34. Arborelius UP, Wretenberg PER, Lindberg F. The effects of armrests and high seat heights on lower-limb joint load and muscular activity during sitting and rising. Ergonomics. 1992;35:1377-91.

35. Alexander NB, Galecki AT, Nyquist LV, Hofmeyer MR, Grunawalt JC, Grenier ML, et al. Chair and bed rise performance in ADL-impaired congregate housing residents. J Am Geriatr Soc. 2000;48:526-33. 